\title{
A multifaceted quality improvement project improves intraoperative redosing of surgical antimicrobial prophylaxis during pediatric surgery
}

\author{
Ashley A. Colletti ${ }^{1}$ (D) | Ellen Wang ${ }^{2}$ | Juan L. Marquez ${ }^{3}$ | Hayden T. Schwenk ${ }^{4}$ | \\ Calida Yeverino $^{5}$ | Paul J. Sharek ${ }^{6}$ | Thomas J. Caruso ${ }^{2}$
}

${ }^{1}$ Division of Pediatric Anesthesia, Department of Anesthesiology and Pain Medicine, Washington and Seattle Children's Hospital, University of Washington, Seattle, Washington

${ }^{2}$ Division of Pediatric Anesthesia,

Department of Anesthesiology,

Perioperative and Pain Medicine, Stanford

University School of Medicine, Stanford,

California

${ }^{3}$ Department of Preventive

Medicine, University of Michigan, School of

Public Health, Ann Arbor

${ }^{4}$ Division of Pediatric Infectious Diseases, Department of Pediatrics, Stanford University School of Medicine, Stanford, California

${ }^{5}$ Department of Pharmacy Services, Lucile Packard Children's Hospital Stanford, Stanford, California

${ }^{6}$ Department of Pediatrics, Washington and Seattle Children's Hospital, University of Washington, Seattle, Washington

\section{Correspondence}

Thomas J. Caruso, Division of Pediatric Anesthesia, Department of Anesthesiology, Perioperative and Pain Medicine, Stanford University School of Medicine, 300 Pasteur Drive H3580, Stanford 94305, CA.

Email: tjcaruso@stanford.edu

Editor: Dean Kurth

\begin{abstract}
Background: Accurate intraoperative antibiotic redosing contributes to prevention of surgical site infections in pediatric patients. Ensuring compliance with evolving national guidelines of weight-based, intraoperative redosing of antibiotics is challenging to pediatric anesthesiologists.

Aims: Our primary aim was to increase compliance of antibiotic redoses at the appropriate time and appropriate weight-based dose to $70 \%$. Secondary aims included a subset analysis of time compliance and dose compliance individually, and compliance based on order entry method of the first dose (verbal or electronic).

Methods: At a freestanding, academic pediatric hospital, we reviewed surgical cases between May 1, 2014, and October 31, 2017 requiring antibiotic redoses. After an institutional change in cefazolin dosing in May 2015, phased interventions to improve compliance included electronic countermeasures to display previous and next dose timing, an alert 5 minutes prior to next dose, and weight-based dose recommendation (September 2015). Physical countermeasures include badge cards, posting of guidelines, and updates to housestaff manual (September 2015). Statistical process control charts were used to assess overall antibiotic redose compliance, time compliance, and dose compliance. The chi-square test was used to analyze group differences.

Results: A total of 3015 antibiotic redoses were administered during 2341 operative cases between May 1, 2014, and October 31, 2017. Mean monthly compliance with redosing was 4.3\% (May 2014-April 2015) and 73\% (November 2015-October 2017) $(P<0.001)$. Dose-only compliance increased from $76 \%$ to $89 \%(P<0.001)$, and timeonly compliance increased from $4.9 \%$ to $82 \%(P<0.001)$. After implementation of countermeasures, electronic order entry compared with verbal order was associated with higher dose compliance, $90 \%$ vs $86 \%(P=0.015)$.

Conclusion: This quality improvement project, utilizing electronic and physical interventions, was effective in improving overall prophylactic antibiotic redosing compliance in accordance with institutional redosing guidelines.
\end{abstract}

\section{KEYWORDS}

antibacterial agents, antibiotic prophylaxis, anti-infective agents, cefazolin, risk factors, surgical wound infection 


\section{1 | INTRODUCTION}

\section{1 | Problem description}

Surgical site infections (SSIs) are a preventable cause of morbidity and mortality in surgical patients. ${ }^{1-3}$ Although prophylactic antibiotic administration has been shown to reduce the incidence of SSIs in both adults and children undergoing surgery, most local and national quality measures report only the appropriate timing and dose of the first antibiotic given prior to incision. ${ }^{2-4}$ However, maintaining adequate inhibitory antimicrobial plasma and tissue levels during surgery depends not just on the initial dose, but also on repeat dosing. ${ }^{1,5}$ Though no randomized control trials have investigated the impact of redosing on the incidence of SSIs, procedure length has been identified as an independent risk factor for developing SSIs. ${ }^{3,6,7}$ Suboptimal redosing patterns of prophylactic antibiotics during longer pediatric surgeries likely contribute to increased risk of SSIs.

\section{2 | Available knowledge}

The Centers for Disease Control (CDC), Infectious Disease Society of America (IDSA), and American Society of Health-System Pharmacists (ASHP) recommend intraoperative redosing when a procedure exceeds one to two half-lives of the antibiotic, when there is major blood loss, or when a patient has extensive burns. ${ }^{3,5}$ The recommended redosing intervals for commonly used antibiotics for surgical prophylaxis were updated in 2013 by the AHSP. ${ }^{5}$ Traditional educational interventions, such as email correspondence or announcements, have been shown to be ineffective in achieving reliable adherence to antibiotic redosing guidelines, with studies reporting compliance below $50 \%$ in some settings. ${ }^{8,9}$ Because redosing guidelines are frequently revised by expert societies, lack of routine compliance measurement results in challenges to reliably adjusting practice habits.

\section{3 | Rationale}

Given the lack of successful practice transformation using traditional methods, coupled with the widespread implementation of anesthesia electronic medical records (EMRs), we utilized clinical informatics to drive a quality improvement project to optimize intraoperative antibiotic redosing after an institutional guideline revision. By leveraging the preexisting standard work of surgeons, pharmacists, and anesthesiologists, and utilizing antibiotic-specific timed alerts in the EMR, we designed a process to improve the accuracy of dose and timing of intraoperative antibiotic redoses.

\section{4 | Specific aims}

The primary aim of this project was to increase appropriate dose and time of intraoperative redosing to $70 \%$ compliance. The secondary aims examined dose and timing of redose independently and also the accuracy of antibiotic redoses when given as electronic compared to verbal orders.

\section{What is already known}

- Maintaining a therapeutic plasma level of prophylactic antibiotics throughout surgical procedures is important for prevention of surgical site infections in pediatric patients.

- Given evolving antibiotic redosing recommendations, traditional methods for ensuring reliable intraoperative redose compliance may be ineffective.

\section{What this article adds}

- Countermeasures integrated into the electronic anesthesia record are effective in improving pediatric anesthesiologists' compliance with institutional guidelines for redosing of surgical prophylactic antibiotics.

\section{2 | MATERIALS AND METHODS}

\subsection{Context}

After the Institutional Review Board approved a waiver for this improvement project, it was initiated by an interdisciplinary quality improvement team at a 311-bed, freestanding academic pediatric hospital with seven operating rooms and 12 off-site procedure locations. Academic faculty, fellows, residents, nurse practitioners, and physician assistants from anesthesia and surgery departments provide care for surgical patients. The institution utilizes an EMR (EPIC).

Inclusion criteria were those patients who underwent procedures at least 2 hours in duration and received at least one intraoperative dose of antibiotic between May 1, 2014, and October 31, 2017, including unscheduled urgent and emergent procedures. Exclusion criteria were those patients who (a) received prophylactic antibiotics requiring greater than 6-hour redosing since they represent a small percentage of antibiotics used for surgical prophylaxis and their redosing recommendations may vary based on organ function or plasma levels, (b) received multiple antibiotics from the same class (such as cefazolin administered at case start followed by cefoxitin), (c) received an antibiotic class change during the procedure, and (d) did not have a weight recorded in the EMR on day of surgery.

\section{2 | Intervention}

\subsection{1 | Current state}

After the AHSP guidelines were updated in 2013, our Antibiotic Stewardship Committee updated institutional guidelines, including a change in cefazolin dose from 20 to $30 \mathrm{mg} / \mathrm{kg}$ in May 2015. While the AHSP guidelines suggest redosing of cefazolin every 4 hours, our Antibiotic Stewardship Committee recommended redosing every 3 hours due to cefazolin half-life of 1.2-2.2 hours to comply with the recommendation to redose every one to two half-lives of 
FIGURE 1 Diagram of key drivers

\begin{tabular}{|c|c|}
\hline \multirow[t]{2}{*}{ Key Drivers } & Interventions \\
\hline & Pop up redosing alert with link to guidelines \\
\hline EMR Optimization & Calculated $\mathrm{mg} / \mathrm{kg}$ and total dose under medication tab \\
\hline \multirow{2}{*}{$\begin{array}{l}\text { Clear communication } \\
\text { of process change }\end{array}$} & $\begin{array}{l}\text { Continuous displayed message of time of last antibiotic } \\
\text { administered in right patient info column }\end{array}$ \\
\hline & Time of next antibiotic dose and frequency in left reminder column \\
\hline \multirow{2}{*}{$\begin{array}{l}\text { Point of care physical } \\
\text { reference guidelines }\end{array}$} & Physician badge with guidelines \\
\hline & Guidelines posted in operating room \\
\hline \multirow{3}{*}{$\begin{array}{l}\text { Reliable process measure } \\
\text { of compliance }\end{array}$} & Updated hospital antimicrobial housestaff manual \\
\hline & $\begin{array}{l}\text { Presented new guidelines at anesthesia and pharmacy division } \\
\text { meetings }\end{array}$ \\
\hline & Developed electronic EMR query for data acquisition \\
\hline
\end{tabular}

FIGURE 2 Electronic countermeasures for antibiotic redosing. Electronic reminder displayed continuously on the left side of the screen with name of antibiotic and time of next indicated redose (A); time of last antibiotic and dose given continuously displayed on the right side of the screen (B); medication administration window that links directly from the alert (D) and suggests doses of last dose given and patient's weight-based dose from guidelines (C); pop-up alert to remind provider $5 \mathrm{~min}$ prior to time of next redose (D)
(A)

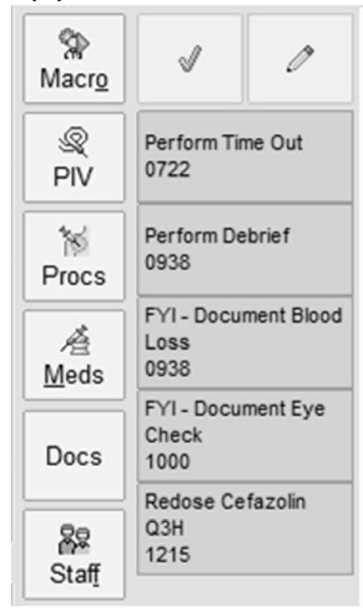

(D)
(B)

- Last $A b x$

Abxc ceFAZolin (ANCEF) inj $1450 \mathrm{mg}$ Given $1400 \mathrm{mg}$ at 0915

Frequency: once

(C)

Minutes since last dose: 189

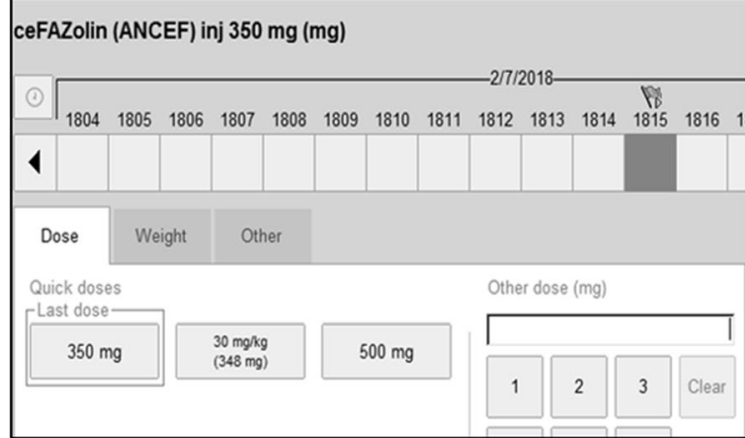

Redose Cefazolin Q3H

1. Antibiotic Redosing Due!

Consider pharmacy consultation for infants and patients with renal dysfunction. an antibiotic and the evidence that SSI rates are significantly higher when a single dose is used for procedures longer than 3 hours in duration. ${ }^{3,5}$ Current state analysis revealed high compliance of appropriate initial preoperative dose and timing due to utilization of electronic order entry and pharmacist preparation. ${ }^{9}$ However, intraoperative antibiotic redose and timing was suboptimal, with a $4 \%$ baseline compliance (May 2014-April 2015). Because subsequent intraoperative doses were prepared and administered by the anesthesiologist, not the pharmacist, a key driver for high compliance was determined to be anesthesiologist accessibility to the new redosing guidelines (Figure 1).

\subsection{2 | Countermeasures}

Electronic countermeasures based on the EMR optimization key driver included the following: (a) An electronic reminder displayed on the left side of the anesthesia record with the name of the last antibiotic administered and time due of the next antibiotic dose based on the revised redosing guidelines (Figure 2A); (b) another electronic display in the right column continuously displayed the antibiotic given and time since the last dose (Figure 2B); (c) clicking on reminders opened a display with the weight-based dose for that antibiotic prepopulated in the anesthesia medication administration record (Figure $2 \mathrm{C}$ ); (d) an additional electronic best practice pop-up alert provided a reminder 5 minutes prior to the time of each redose (Figure 2D). These electronic countermeasures were implemented in September 2015

Physical countermeasures based on the point-of-care physical reference guidelines key driver included the following: (a) distribution of antibiotic redose guideline badge cards to anesthesia providers, (b) posting of redosing guidelines on the operating room wall behind the anesthesia supply cart, and (c) updating the hospital's 
antimicrobial house staff manual. These displays included the redosing frequency, weight-based dose, and maximum dose for common surgical prophylactic antibiotics. These interventions were phased, concluding in September 2015.

\subsection{3 | Implementation}

To ensure clear communication of the process change, another key driver, updates made by the Antibiotic Stewardship Committee to the surgical antibiotic prophylaxis guidelines were emailed out to the perioperative team and presented at interdisciplinary meetings (July 2015). A report of antibiotic electronic order entry compliance was emailed monthly to surgical chiefs after implementation. Incremental feedback of improvement efforts was provided to department members in the form of statistical process control (SPC) charts throughout the intervention, which was developed in order to address the final key driver, a reliable process measure of compliance.

\subsection{4 | Measures}

Patient characteristics (age, weight), order entries, and medication administration records were electronically obtained from the EMR. The primary outcome was compliance with administration of antibiotic redose at both the appropriate time ( \pm 15 minutes) and appropriate dose, defined as the patient's calculated weight-based dose $( \pm 10 \%)$ until maximum dose was reached. Maximum dose and redose interval adjustments were made for certain antibiotics in neonates (Table 1). A dose was considered compliant if it first met criteria for appropriate time and then for weight-based dose. Secondary outcomes examined were (a) time compliance only, (b) dose compliance only, and (c) compliance between redoses after the first dose was ordered via electronic order entry compared to those administered after verbal order between surgeon and anesthesiologist. We set a goal of achieving and maintaining $70 \%$ compliance for antibiotic redoses.

\section{3 | Analysis}

A SPC chart was used to display compliance of antibiotic redose administration time and dose for all cases by month. Center lines $(\mathrm{CL})$, representing average compliance, were displayed to demonstrate responses to countermeasures. Upper and lower control limits were included on the charts, and breaks in the $\mathrm{CL}$ were at times of special cause variation attributed to nonrandom conditions per the Western Electric Rules. ${ }^{10,11}$ There are several rules for special cause variation, and the basic four are as follows: Rule 1-a point outside of three standard deviations from the same side of the centerline is not due to common cause variation; Rule 2-two of three consecutive points that fall beyond two standard deviations on the same side of the centerline are not due to common cause variation; Rule 3-four of five consecutive points that fall beyond one standard deviation on the same side of the centerline are not due to common cause variation; and Rule 4-nine consecutive points that fall on the same side of the centerline are not due to common cause variation. Data were collected and analyzed using Microsoft Excel, and statistical analyses were performed using $\mathrm{R}$ version 3.4.3. Chi-square tests were used to determine statistical significance of group differences, with $P<0.05$ considered statistically significant. The pre-intervention group was comprised of cases between May 2014 through July 2015, and the post intervention group included August 2015 through October 2017.

\subsection{Ethical considerations}

No notable conflicts of interest were identified in the carrying out of this project. Ethically, we did consider that improved reliance on electronic reminders for antibiotic redosing could potentially prompt a provider to administer too-frequent dosing of an antibiotic in patients who require an alternative dosing schedule, such as those with compromised renal function, since pharmacists do not review intraoperative medications prior to administration, and that this could result in potential harm.

\section{3 | RESULTS}

Between May 1, 2014, and October 31, 2017, there were 2685 operative cases potentially requiring redoses. After application of exclusion criteria (93 cases were spurious data that did not meet inclusion criteria, 90 cases lacked weight, 65 cases received antibiotic redoses greater than 6 hours apart, 41 cases had an intraoperative antibiotic class change, 11 cases received antibiotics from the same class, and two procedures lacked a start or end time), 2338 out of 17735 cases (13\%) were included in the analysis and there were 2952 antibiotic redoses administered in the included cases.

\section{1 | Primary Aim}

\subsection{1 | Overall antibiotic redose compliance}

Mean percentage monthly compliance with antibiotic redosing at both the correct time and correct dose is represented by the $\mathrm{CL}$ (Figure 3). The mean compliance began at 4.3\% (May 2014-April 2015 ) and rose to $73 \%$, with the establishment of a new CL (CL \#3, November 2015-October 2017), $P<0.001$, surpassing our shortterm target of $70 \%$ of antibiotic redoses given at the correct time and correct dose.

\subsection{Secondary Aims 1 and 2}

\subsection{1 | Dose compliance only and time compliance only}

Dose compliance for any antibiotic redose administered, regardless of timeliness, increased from 76\% (CL\#1) to 89\% (CL \#3), $P<0.001$, 
TABLE 1 Antibiotic redose guidelines for surgical prophylaxis as determined by our institution's antibiotic stewardship committee

\begin{tabular}{|c|c|c|c|}
\hline Antibiotic & Dose (mg/kg) & Max dose & Age-based OR redose \\
\hline \multirow[t]{2}{*}{ Ampicillin } & \multirow[t]{2}{*}{50} & \multirow[t]{2}{*}{$2 \mathrm{~g}$} & <14 d OR <2 kg: $6 \mathrm{~h}$ \\
\hline & & & $>15 \mathrm{~d}$ AND >2 kg: $3 \mathrm{hrs}$ \\
\hline \multirow[t]{2}{*}{ Ampicillin-Sulbactam } & \multirow[t]{2}{*}{50} & \multirow[t]{2}{*}{$2 \mathrm{~g}$} & $<1 \mathrm{mo}$ : contact pharmacy \\
\hline & & & $>1 \mathrm{mo}: 3 \mathrm{~h}$ \\
\hline \multirow[t]{2}{*}{ Cefazolin } & \multirow[t]{2}{*}{30} & $<120 \mathrm{~kg}: 2 \mathrm{~g}$ & $<7$ d OR <2 kg: $6 \mathrm{~h}$ \\
\hline & & $>120 \mathrm{~kg}: 3 \mathrm{~g}$ & $>7 \mathrm{~d}$ AND >2 kg: $3 \mathrm{~h}$ \\
\hline \multirow[t]{3}{*}{ Cefotaxime } & \multirow[t]{3}{*}{50} & \multirow[t]{3}{*}{$2 \mathrm{~g}$} & $<7$ d OR <2 kg: 8 h \\
\hline & & & $>7 \mathrm{~d}$ AND $2 \mathrm{~kg}: 6 \mathrm{~h}$ \\
\hline & & & $>1 \mathrm{mo}: 3 \mathrm{~h}$ \\
\hline \multirow[t]{2}{*}{ Cefoxitin } & \multirow[t]{2}{*}{40} & \multirow[t]{2}{*}{$2 \mathrm{~g}$} & $<1 \mathrm{mo}: 3 \mathrm{~h}$ \\
\hline & & & $>1 \mathrm{mo}: 2 \mathrm{~h}$ \\
\hline \multirow[t]{2}{*}{ Clindamycin } & $<1 \mathrm{mo:} 5$ & \multirow[t]{2}{*}{$900 \mathrm{mg}$} & $<7 \mathrm{~d}$ OR $<2 \mathrm{~kg}: 12 \mathrm{~h}$ \\
\hline & >1 mo: 10 & & $>7 \mathrm{~d} \mathrm{OR}>2 \mathrm{~kg}: 6 \mathrm{~h}$ \\
\hline
\end{tabular}

and time compliance for any antibiotic redose, regardless of dose administered, increased from $4.9 \%$ (CL \#1) to $82 \%(C L \# 3), P<0.001$ (Figure 4A, 4B).

\subsection{Secondary Aim 3}

\subsection{1 | Electronic vs verbal order compliance}

For 1362 redoses, an electronic order was placed prior to the operation by the surgeon for the initial antibiotic dose to be prepared by the pharmacist. The remaining 1590 redoses were administered to patients after the surgeon verbally gave the order to the anesthesiologist. Pre-intervention, prior to July 2015, electronic order entry was associated with significantly higher compliance for time and dose combined, time only, and dose only, $P<0.05$. Postintervention, electronic order entry impacted only the dose of antibiotic given, with $90 \%$ compliance observed for redoses given after an electronic order, compared with $86 \%$ compliance for those ordered verbally $(P=0.015)$.

\section{4 | DISCUSSION}

\section{1 | Summary}

To the authors' knowledge, this is the first description of a quality improvement project to increase compliance with appropriate intraoperative antibiotic redosing guidelines for pediatric surgeries. This project demonstrates the effectiveness of a multifaceted intervention, including timed electronic alerts displayed on the anesthesia provider's EMR and cognitive aids, resulting in an 18-fold increase in redose compliance. This is significant given the morbidity associated with SSIs and the role that timely and accurately dosed antibiotics have in maintaining therapeutic plasma levels. ${ }^{5}$ By leveraging standard workflow, we successfully created a process that did not just result in a temporary compliance improvement but demonstrated sustainability for nearly 2 years.

\section{2 | Interpretation}

In order to determine whether there were differences between which factors were contributing to compliance (dose or time), we also examined intraoperative compliance independently. Timing of redoses had a larger increase in compliance, likely due to the electronic and physical countermeasures described. The weightbased dose compliance increased significantly but without the magnitude of the timing increase in compliance, indicating that timing was a more significant barrier to compliance. Dose compliance of subsequent redoses was higher when an electronic order was placed for the first antibiotic dose. The EMR alerts reminded providers of the correct antibiotic dose and appropriate time for redosing, regardless of whether the first dose was ordered verbally or electronically. Also, the medication administration popup included weight-based dosing guidance alongside the previous dose.

Surgeons perform nearly one million pediatric operations each year in the United States, with a reported SSI incidence of $2 \%-5 \%{ }^{12}$ Given that approximately half of surgical site infections are preventable with appropriate antibiotic prophylaxis, optimization of intraoperative antibiotic redosing has the potential to reduce morbidity and mortality. ${ }^{3,10,13,14}$ Although duration of surgery greater than 2 hours has been identified as an independent factor for SSI, the Surgical Care Improvement Project (SCIP) and CDC guidelines focus only on reporting appropriate first, preoperative dose of antibiotic, not subsequent intraoperative redoses. ${ }^{6,7}$ Because bolus dosing of an antibiotic results in a peak and trough of plasma levels, there is a high risk of falling below the minimal inhibitory concentration (MIC) in longer surgeries when redosing is delayed or neglected, and current evidence does demonstrate that cefazolin levels fall below the MIC prior to 4 hours after a first dose. ${ }^{13}$ Maintaining the therapeutic plasma and tissue levels required to prevent infection in surgeries of this duration requires appropriate redosing based on the half-life of antibiotic given. 


\section{Antibiotic Redose Compliance (Dose \& Time) by Month}

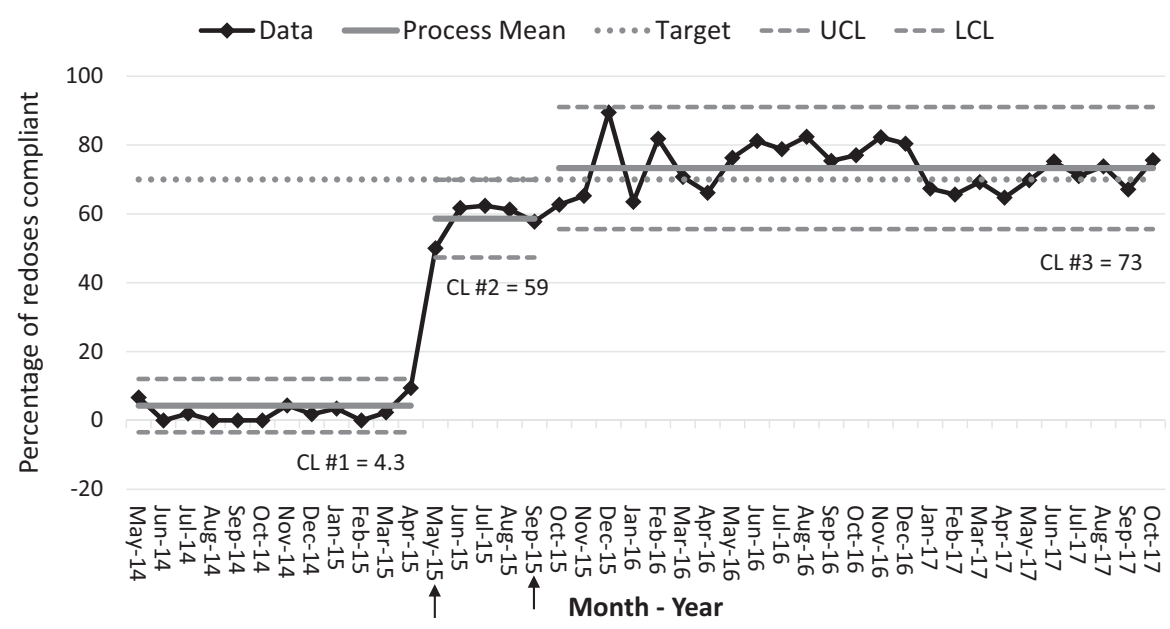

FIGURE 3 Statistical process control chart for overall antibiotic redose compliance by month, defined as by administration of the correct weightbased dose at the indicated time for redosing. Arrows on $x$-axis represent institutional change in redosing guidelines (May 2015) and completion of phased interventions, including physical and electronic countermeasures (September 2015)
(A) Antibiotic Redose Compliance (Time Only) by Month

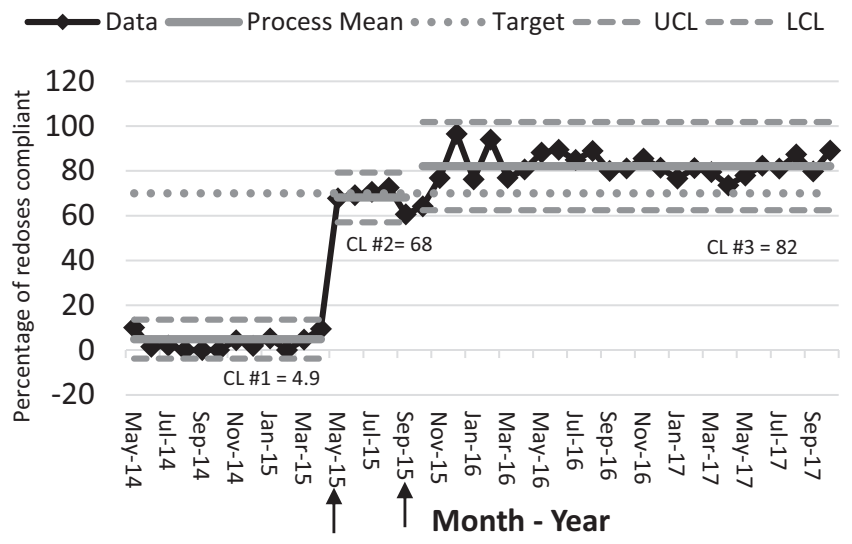

(B) Antibiotic Redose Compliance (Dose Only) by Month

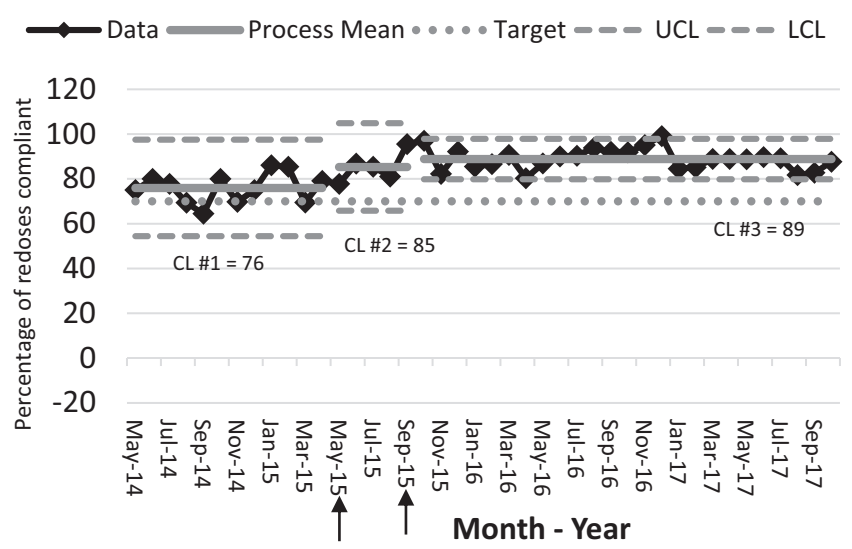

FIGURE 4 Antibiotic redose compliance by time only (A) and dose only (B). Arrows on $x$-axis represent institutional change in redosing guidelines (May 2015) and completion of phased interventions, including physical and electronic countermeasures (September 2015)

\section{3 | Limitations}

This project had several limitations. First, as a quality improvement project with multiple countermeasures, it is possible that increased provider awareness and altered practice habits contributed to the results and not the countermeasures described. However, given the temporal relationship between countermeasure deployment and increase in compliance, we find this to be unlikely. Second, compliance with utilization of individual countermeasures was not measured. It is unknown which countermeasures contributed more to the results since multiple countermeasures were initiated simultaneously. Third, our electronic reporting algorithm did not provide nontemporal indications for antibiotic redosing, such as large-volume blood loss or burn physiology, which can alter plasma levels of an antibiotic.

While our antibiotic redose compliance reached our stepwise aim of $70 \%$ compliance, the long-term goal of this quality improvement initiative is full compliance. Future efforts will seek to determine unknown barriers to achieving full compliance and could explore an association between low plasma levels of antibiotic with increased infection risk. Next steps should consider robust alerts with the ability to open in front of any window on the anesthesia computer screen or adding an audible alert when a redose is due. Given the high morbidity associated with SSIs, monitoring of intraoperative antibiotic redosing compliance should be considered by practitioners seeking to reduce the risk of SSIs.

\section{CONFLICT OF INTEREST}

The authors have no disclosures.

\section{ORCID}

Ashley A. Colletti (iD https://orcid.org/0000-0002-6961-6546

Thomas J. Caruso iD https://orcid.org/0000-0002-0723-5262 


\section{REFERENCES}

1. Dellinger EP, Gross PA, Barrett TL, et al. Quality standard for antimicrobial prophylaxis in surgical procedures. Infect Control Hosp Epidemiol. 1994;15:182-188.

2. Bratzler DW, Hunt DR. The surgical infection prevention and surgical care improvement projects: national initiatives to improve outcomes for patients having surgery. Clin Infect Dis. 2006;43: 322-330.

3. Berríos-Torres SI, Umscheid CA, Bratzler DW, et al. Centers for disease control and prevention guideline for the prevention of surgical site infection, 2017. JAMA Surg. 2017;152:784-791.

4. Khoshbin A, So JP, Aleem IS, Stephens D, Matlow AG, Wright JG. Antibiotic prophylaxis to prevent surgical site infections in children: a prospective cohort study. Ann Surg. 2015;262:397-402.

5. Bratzler DW, Dellinger EP, Olsen KM, et al. Clinical practice guidelines for antimicrobial prophylaxis in surgery. Am J Health Syst Pharm. 2013;70:195-283.

6. Leong G, Wilson J, Charlett A. Duration of operation as a risk factor for surgical site infection: comparison of English and US data. J Hosp Infection. 2006;63:255-262.

7. Kasatpibal N, Whitney JD, Dellinger EP, et al. Failure to Redose Antibiotic Prophylaxis in Long Surgery Increases Risk of Surgical Site Infection. Surg Infect. 2017;18:474-484

8. Knox MC, Edye M. Educational antimicrobial stewardship intervention ineffective in changing surgical prophylactic antibiotic prescribing. Surg Infect (Larchmt). 2016;17:224-228.
9. Caruso TJ, Wang E, Schwenk HT, et al. A quality improvement initiative to optimize dosing of surgical antimicrobial prophylaxis. Pediatr Anesth. 2017;27:702-710.

10. The Western Electric Company. Statistical Quality Control Handbook. Easton, PA: The Mack Printing Company; 1958.

11. Kurth CD. Introducing quality improvement. Pediatr Anesth. 2013;23(7):569-570.

12. Somme S, Bronsert M, Morrato E, Ziegler M. Frequency and variety of inpatient pediatric surgical procedures in the United States. Pediatrics. 2013;132:e1466-1472.

13. Morse J, Blackburn L, Hannam JA, Voss L, Anderson BJ. Compliance with perioperative prophylaxis guidelines and the use of novel outcome measures. Pediatr Anesth. 2018;28:686-693.

14. Caruso TJ, Wang EY, Colletti AA, Sharek PJ. Intraoperative antibiotic redosing compliance and the extended postoperative recovery period: often overlooked areas that may reduce surgical site infections. Pediatr Anesth. 2019;29:290-291.

How to cite this article: Colletti AA, Wang E, Marquez JL, et al. A multifaceted quality improvement project improves intraoperative redosing of surgical antimicrobial prophylaxis during pediatric surgery. Pediatr Anesth. 2019;29:705-711. https://doi.org/10.1111/pan.13651 